\title{
BMJ Open Microscopic decompressive laminectomy versus percutaneous endoscopic decompressive laminectomy in patients with lumbar spinal stenosis: protocol for a systematic review and meta-analysis
}

\author{
Rong Wang, ${ }^{1}$ Xiuxia Li, ${ }^{2,3}$ Xiaogang Zhang, ${ }^{1}$ Daping Qin, ${ }^{1}$ Guodong Yang, ${ }^{1}$
} Guodong Gao, ${ }^{1}$ Hua Zhang (iD ${ }^{1}$

To cite: Wang $\mathrm{R}$, Li X Zhang $X$, et al. Microscopic decompressive laminectomy versus percutaneous endoscopic decompressive laminectomy in patients with lumbar spinal stenosis: protocol for a systematic review and meta-analysis. BMJ Open 2020;10:e037096. doi:10.1136/ bmjopen-2020-037096

- Prepublication history and additional material for this paper are available online. To view these files, please visit the journal online (http://dx.doi. org/10.1136/bmjopen-2020037096).

RW and $\mathrm{XL}$ are joint first authors.

Received 01 February 2020 Revised 30 June 2020 Accepted 13 August 2020

Check for updates

(C) Author(s) (or their employer(s)) 2020. Re-use permitted under CC BY-NC. No commercial re-use. See rights and permissions. Published by BMJ.

For numbered affiliations see end of article.

Correspondence to Professor Hua Zhang; major1982@163.com and Dr Guodong Gao; goodrunner@126.com

\section{ABSTRACT}

Introduction Lumbar spinal stenosis (LSS) is a common lumbar degenerative disease in the elderly, usually requiring surgery if conservative treatment fails. Microscopic decompressive laminectomy (MDL) and percutaneous endoscopic decompressive laminectomy (PEDL) have been widely used to treat LSS. This study aims to provide a protocol for the evaluation and comparison of the efficacy, safety and applicability between MDL and PEDL.

Methods and analysis We will search for randomised controlled trials (RCTs) comparing MDL and PEDL for treating LSS from inception to December 2019 in the following databases: PubMed, The Cochrane Library, Web of Science, Embase and China Biology Medicine. The quality of included studies will be assessed using the risk of bias tool recommended by the Cochrane Handbook 5.2.0. Subsequently, a meta-analysis will be performed using RevMan 5.3 software.

Ethics and dissemination Given the nature of this study, no ethical approval will be required. The protocol will be disseminated via a peer-reviewed journal. PROSPERO registration number CRD42020164765.

\section{INTRODUCTION}

Lumbar spinal stenosis (LSS) occurs predominantly in the elderly, with an incidence rate of $10.3 \%-11.2 \%$ in individuals aged over 70 years. It is divided into central spinal stenosis, lateral crypt stenosis and neural root canal stenosis. LSS is mainly caused by congenital spinal dysplasia or acquired factors, such as bone hyperplasia, disc herniation, ligament hypertrophy, spinal trauma, lumbar spondylolisthesis and iatrogenic LSS. A typical symptom of LSS is neurogenic intermittent claudication; in severe cases, patients may also show symptoms of cauda equina syndrome, which requires emergency surgery. ${ }^{1-4}$
Strengths and limitations of this study

- This study will first systematically evaluate the efficacy, safety and applicability of microscopic decompressive laminectomy and percutaneous endoscopic decompressive laminectomy.

- The research results will provide a reference for clinicians to choose an appropriate minimally invasive surgery for lumbar spinal stenosis.

- This study provides improved clinical applicability and comparability of derived results through subgroup analysis.

- The results of this study may change and need to be updated when new high-quality randomised controlled trials are reported.

- We only include English and Chinese literatures, which may lead to a selection bias.

The gold standard procedure for LSS is open decompressive laminectomy, but in recent years, minimally invasive decompressive laminectomy (MIDL) procedures such as microscopic decompressive laminectomy (MDL) and percutaneous endoscopic decompressive laminectomy (PEDL) using smaller incisions have become more popular due to reportedly satisfactory results. ${ }^{5-8} \mathrm{~A}$ number of randomised controlled trials (RCTs) comparing MDL and PEDL for LSS have been published, ${ }^{6-10}$ but high-quality systematic reviews (SRs) are lacking. Therefore, this study uses a meta-analysis method to systematically evaluate and compare the efficacy, safety and applicability between MDL and PEDL provide a basis for spinal surgeons to choose an appropriate surgical procedure. 


\section{METHODS}

\section{Inclusion criteria}

Type of study

Only RCTs comparing MDL with PEDL for treating LSS will be included in this SR. Patients should be randomly assigned into MDL and PEDL groups. The language of the literature will be limited in English or Chinese.

\section{Participants}

This study includes patients diagnosed with LSS according to the criteria defined by the North American Spine Society. ${ }^{1}$ Patients with lumbar surgery history, epidural injection history, specific or non-specific infection and tumour will be excluded.

\section{Interventions}

The MDL procedure is briefly described as follows: after confirming the target level, a small skin incision $(3-4 \mathrm{~cm})$ is made approximately $1.5 \mathrm{~cm}$ lateral to the spinous process, placing the tubular retractor on a series of tubular dilators through the intermuscular space for retraction. Following this, laminectomy, flavectomy and partial facet resection are performed to achieve spinal canal decompression with the aid of a microscope. Further, contralateral decompression can be performed by tilting the operating table and microscope. ${ }^{611-13}$

The PEDL procedure is briefly described as follows: a working sheath and working-channel endoscope are inserted through a $1 \mathrm{~cm}$ incision, $0.5-1 \mathrm{~cm}$ lateral to the spinous process. Instruments for laminectomy are inserted through the working channel. An endoscopic laminectomy is identical to a microscopic laminectomy but under continuous saline irrigation. The surgical field is visualised using a monitor system. ${ }^{689}$

Cointervention, conventional open techniques using loupe magnification and lumbar decompression combined with lumbar interbody fusion will be excluded.

\section{Outcomes}

The primary outcomes will include lower back and leg pain intensity measurement via a Visual Analogue Scale (VAS) or a Numerical Rating Scale (NRS) within 2 weeks after surgery. Oswestry Disability Index (ODI) and European Quality of Life-5 Dimensions (EQ-5D) score will be collected within 1 year after surgery. Secondary outcomes will include operation time, intraoperative blood loss, postoperative drainage and serum creatine phosphokinase level at 2 days after surgery, and adverse events (eg, dural tear, nerve root injury, incomplete decompression, reoperation and epidural hematoma).

\section{Data sources}

We will search PubMed, The Cochrane Library, Web of Science, Embase and China Biology Medicine databases from inception to December 2019.

\section{Search strategy}

We will use the following English search terms to build our search strategy: (( (spin* OR lumb* OR "nerve root canal" OR "lateral recess" OR "lateral crypt") AND stenos*) OR "intermittent claudication" OR LSS OR DLSS OR LSCS OR LCS) AND (decompressi* OR minimally OR micro* OR laminectom* OR MISS OR fullendoscop* OR endoscop*) AND (random* OR blind* OR "controlled clinical trial*"). Besides, we will search for Medical Subject Headings (MeSH) terms as follows: ("Spinal Canal" [Mesh] OR "Lumbar Vertebrae"[Mesh] OR "Spinal Diseases" [Mesh] OR "Constriction, Pathologic"[Mesh] OR "Spinal Stenosis"[Mesh] OR "Lumbar Stenosis, Familial" [Supplementary Concept] OR "Spinal Osteophytosis"[Mesh] OR "Spondylosis"[Mesh]) AND ("Decompression"[Mesh] OR "Decompression, Surgical"[Mesh] OR "Minimally Invasive Surgical Procedures"[Mesh] OR "Microsurgery"[Mesh] OR "Endoscopes"[Mesh] OR "laminectomy"[Mesh]) AND ("Randomized Controlled Trials as Topic"[Mesh] OR "Randomized Controlled Trial"[Publication Type] OR "Controlled Clinical Trial" [Publication Type] OR "Controlled Clinical Trials as Topic"(Mesh) OR "blindness"(Mesh)) (see online supplemental file).

\section{Study selection and data extraction}

After selecting studies according to the above-mentioned inclusion criteria, two independent reviewers will read the full texts and extract the data. Disagreements between reviewers will be resolved by consultation with a third researcher. If the data included in the study are incomplete or the data are reported in the form of a graph, we will contact the author via email to obtain the original data. If the author cannot be contacted or refuses to provide the data, we will remove the study from the metaanalysis and give a statistical description in the Results section.

\section{Quality assessment of included studies}

The quality of the RCTs will be assessed using the risk of bias (RoB) tool recommended by the Cochrane Handbook V.5.2.0 (Cochrane Collaboration, London, UK). This assessment is performed considering six aspects: (1) random sequence generation; (2) allocation concealment; (3) blinding of participants and outcome assessors; (4) incomplete outcome data; (5) selective reporting and (6) other bias. The RoB in each domain is classified as low risk, high risk or unclear. If the RoBs of the studies included in the meta-analysis differ, we will conduct a hierarchical analysis based on the RoB to show readers the different results under different RoBs.

\section{Subgroup analysis}

To reduce the effect of clinical heterogeneity between studies on the conclusions of the meta-analysis and to observe whether the clinical efficacy and incidence of complications in the two procedures are different in different areas of stenosis (central or lateral recess stenosis, foraminal stenosis), scopes of decompression (unilateral or bilateral decompression) and levels of 
decompression (single-level or multilevel decompression), a subgroup analysis will be conducted.

\section{Data synthesis and analysis}

We will use RevMan 5.3 software (Cochrane Collaboration) to perform the meta-analysis. If more than two studies include primary outcomes, a meta-analysis will be conducted. Weighted mean difference or standard mean difference with $95 \%$ CI will be calculated for continuous data. The Higgins $\mathrm{I}^{2}$ test will be used to assess heterogeneity, with a significance level set at $25 \%$. A fixed-effect model will be used in the case of low heterogeneity $\left(\mathrm{I}^{2} \leq 25 \%\right)$; otherwise, a random effects model $\left(\mathrm{I}^{2}>25 \%\right)$ will be used. ${ }^{14}$ Meta-regression (if more than 10 studies are included) or sensitivity analysis will be applied to explore the source of heterogeneity. If the $\mathrm{I}^{2}$ value of the combined results is greater than $75 \%$, we will abandon the meta-analysis and only give a general statistical description of the research results. ${ }^{14}$

The minimal clinically important difference (MCID) is defined as 'the smallest difference in a score that is considered to be worthwhile or important'. ${ }^{15}$ Thus, if the metaanalysis results reach or exceed this score, the results will be considered as meaningful and worthwhile. The MCID scores of the primary outcomes are regarded as follows: VAS, 3.0 ${ }^{13}{ }^{16}$; NRS, 2.0 ${ }^{17}$; ODI, $12.0^{13}$ and EQ-5D, 0.24. ${ }^{16}$

Finally, a funnel plot will be constructed following Egger's test to evaluate publication bias.

\section{Quality of evidence}

The grading of recommendations, assessment, development and evaluation (GRADE) will be used to assess the quality of evidence for all outcomes, including the following: RoB, inaccuracy, inconsistency, indirectness and publication bias. The results of assessment will be graded under four levels: very low, low, moderate and high levels. ${ }^{18}$

\section{Patient and public involvement}

Patients and the public were not involved in the design or planning of this SR protocol.

\section{DISCUSSION}

Evidence-based medicine has shown that compared with conventional open decompression laminectomy, MIDL is more accurate, with less intraoperative bleeding, less postoperative pain, faster body function recovery and shorter hospitalisation duration. ${ }^{19}{ }^{20}$ However, because of some limitations of MIDL (eg, difficulty in instrument manipulation, poor visualisation and long learning cycle), which results in inadequate decompression, nerve damage and prolonged operation time, the success rate of MIDL is still controversial. ${ }^{620}{ }^{21}$ Although MIDL has many issues to be resolved, with continuous improvement of equipment and the gradual maturation of surgical technology, the advantages of MIDL over the conventional open decompressive laminectomy are increasing, and it is expected to become the new technical standard. ${ }^{20}$ 22-24

We will perform this study in strict accordance with the Cochrane Handbook for Systematic Reviews of Interventions, and report in accordance with the Preferred Reporting Items for Systematic Reviews and Meta-Analyses guidelines. We want to conduct this SR to comprehensively evaluate MDL and PEDL and compare them, and provide some suggestions for its reasonable and effective clinical application.

\section{TRIAL STATUS}

- Preliminary searches: started.

- Piloting of the study selection process: not started.

- Formal screening: not started.

- Data extraction: not started.

- RoB assessment: not started.

- Data analysis: not started.

\section{ETHICS AND DISSEMINATION}

Because this is an academic review of the published literature, no ethical approval will be required. This protocol will be submitted to a recognised journal for publication and to presentations at national and international conferences.

\section{Author affiliations}

${ }^{1}$ Department of Spine Surgery, Affiliated Hospital of Gansu University of Chinese Medicine, Lanzhou, Gansu, China

${ }^{2}$ Evidence-Based Social Science Research Center, School of Public Health, Lanzhou University, Lanzhou, Gansu, China

${ }^{3}$ Key Laboratory of Evidence Based Medicine and Knowledge Translation of Gansu Province, Lanzhou, Gansu, China

Acknowledgements We would like to express our gratitude to teachers of Evidence-Based Social Science Research Center of Lanzhou University for their methodological help. We would also thank the library of Lanzhou University for the access to the different databases and for retrieving of full texts of articles.

Contributors Conceptualization: $\mathrm{HZ}$ and RW. Data curation: GG and GY. Methodology: XL. Project administration: XZ. Protocol draft: RW. Software: GG and DQ. Study design: HZ, RW and XL. Validation: XZ. Writing (original draft): RW. Writing (review and editing): XL. All authors reviewed and approved the manuscript in its current form.

Funding The authors have not declared a specific grant for this research from any funding agency in the public, commercial or not-for-profit sectors.

Competing interests None declared.

Patient and public involvement Patients and/or the public were not involved in the design, or conduct, or reporting, or dissemination plans of this research.

Patient consent for publication Not required.

Provenance and peer review Not commissioned; externally peer reviewed.

Open access This is an open access article distributed in accordance with the Creative Commons Attribution Non Commercial (CC BY-NC 4.0) license, which permits others to distribute, remix, adapt, build upon this work non-commercially, and license their derivative works on different terms, provided the original work is properly cited, appropriate credit is given, any changes made indicated, and the use is non-commercial. See: http://creativecommons.org/licenses/by-nc/4.0/.

ORCID iD

Hua Zhang http://orcid.org/0000-0001-7688-8462 


\section{REFERENCES}

1 Kreiner DS, Shaffer WO, Baisden JL, et al. An evidence-based clinical guideline for the diagnosis and treatment of degenerative lumbar spinal stenosis (update). Spine J 2013;13:734-43.

2 Aleem IS, Drew B. Cochrane in CORR $®$ : surgical versus nonsurgical treatment for lumbar spinal stenosis. Clin Orthop Relat Res 2017;475:2632-7.

3 Matz PG, Meagher RJ, Lamer T, et al. Guideline summary review: an evidence-based clinical guideline for the diagnosis and treatment of degenerative lumbar spondylolisthesis. Spine J 2016;16:439-48.

4 Trigg SD, Devilbiss Z. Spine conditions: lumbar spinal stenosis. FP Essent 2017;461:21-5.

5 Mobbs RJ, Li J, Sivabalan P, et al. Outcomes after decompressive laminectomy for lumbar spinal stenosis: comparison between minimally invasive unilateral laminectomy for bilateral decompression and open laminectomy: clinical article. J Neurosurg Spine 2014;21:179-86.

6 Park S-M, Park J, Jang HS, et al. Biportal endoscopic versus microscopic lumbar decompressive laminectomy in patients with spinal stenosis: a randomized controlled trial. Spine J 2020;20:156165.

7 Fujimoto T, Taniwaki T, Tahata S, et al. Patient outcomes for a minimally invasive approach to treat lumbar spinal canal stenosis: is microendoscopic or microscopic decompressive laminotomy the less invasive surgery? Clin Neurol Neurosurg 2015;131:21-5.

8 Kang T, Park SY, Kang CH, et al. Is biportal technique/endoscopic spinal surgery satisfactory for lumbar spinal stenosis patients?: a prospective randomized comparative study. Medicine 2019;98:1-7.

9 Park S-M, Kim G-U, Kim H-J, et al. Is the use of a unilateral Biportal endoscopic approach associated with rapid recovery after lumbar decompressive laminectomy? A preliminary analysis of a prospective randomized controlled trial. World Neurosurg 2019;128:e709-18.

10 Ruetten S, Komp M, Merk H, et al. Surgical treatment for lumbar lateral recess stenosis with the full-endoscopic interlaminar approach versus conventional microsurgical technique: a prospective, randomized, controlled study. J Neurosurg Spine 2009;10:476-85.

11 Dohzono S, Toyoda $\mathrm{H}$, Matsumura A, et al. Clinical and radiological outcomes after microscopic bilateral decompression via a unilateral approach for degenerative lumbar disease: minimum 5-year followup. Asian Spine J 2017;11:285-93.
12 Rahman M, Summers LE, Richter B, et al. Comparison of techniques for decompressive lumbar laminectomy: the minimally invasive versus the "classic" open approach. Minim Invasive Neurosurg 2008;51:100-5.

13 Alimi M, Hofstetter CP, Pyo SY, et al. Minimally invasive laminectomy for lumbar spinal stenosis in patients with and without preoperative spondylolisthesis: clinical outcome and reoperation rates. $J$ Neurosurg 2015;22:339-52.

14 Higgins JPT, Thompson SG, Deeks JJ, et al. Measuring inconsistency in meta-analyses. BMJ 2003;327:557-60.

15 Beaton DE, Boers M, Wells GA, et al. Many faces of the minimal clinically important difference (MCID): a literature review and directions for future research. Curr Opin Rheumatol 2002;14:109-14.

16 Parker SL, Adogwa O, Mendenhall SK, et al. Determination of minimum clinically important difference (MCID) in pain, disability, and quality of life after revision fusion for symptomatic pseudoarthrosis. Spine J 2012;12:1122-8.

17 Ulrich NH, Kleinstück F, Woernle CM, et al. Clinical outcome in lumbar decompression surgery for spinal canal stenosis in the aged population: a prospective Swiss multicenter cohort study. Spine 2015;40:415-22.

18 Puhan MA, Schunemann HJ, Murad MH, et al. A grade Working group approach for rating the quality of treatment effect estimates from network meta-analysis. BMJ 2014;349:g5630-10.

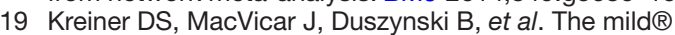
procedure: a systematic review of the current literature. Pain Med 2014;15:196-205.

20 Phan K, Mobbs RJ. Minimally invasive versus open laminectomy for lumbar stenosis: a systematic review and meta-analysis. Spine 2016;41:E91-100.

21 Nerland US, Jakola AS, Solheim O, et al. Minimally invasive decompression versus open laminectomy for central stenosis of the lumbar spine: pragmatic comparative effectiveness study. BMJ 2015;350:1-9.

22 Ikuta K. Microendoscopic Laminotomy for lumbar spinal stenosis. Tech Orthop 2011;26:213-5.

23 Polikandriotis JA, Hudak EM, Perry MW. Minimally invasive surgery through endoscopic laminotomy and foraminotomy for the treatment of lumbar spinal stenosis. J Orthop 2013;10:13-16.

24 Mobbs R, Phan K. Minimally invasive unilateral laminectomy for bilateral decompression. JBJS Essent Surg Tech 2017;7:e9-8. 